Notre Dame Journal of Formal Logic

Volume 36, Number 1, Winter 1995

\title{
Belief Revision and Verisimilitude
}

\author{
MARK RYAN and PIERRE-YVES SCHOBBENS
}

\begin{abstract}
The Egli-Milner power-ordering is used to define verisimilitude orderings on theories from preference orderings on models. The effects of the definitions on constraints such as stopperedness and soundness are explored. Orderings on theories are seen to contain more information than orderings on models. Belief revision is defined in terms of both types of orderings, and conditions are given which make the two notions coincide.
\end{abstract}

1 Introduction Belief revision and verisimilitude involve very similar notions. In both cases we wish to select a theory according to some notion of 'closeness' to a given theory. In the case of belief revision, we are given a sentence, and the selection is from all the theories that contain the sentence. In the case of verisimilitude, the given theory represents the truth, and we are directly given the family of theories from which to select. But the criterion is the same: we seek a theory in the family of theories which is closest to the given theory.

From a technical perspective, however, the two topics have received different treatments. Our aim in this paper is to formalize the intuitive relations between the two topics by providing maps which define one concept in terms of the other. We will also consider the topic of preference relations in our analysis. Preference relations (cf. Shoham [17], Kraus, Lehman, and Magidor [5], and Makinson 8]) were defined to give a semantics to default reasoning. Since the relationship between default reasoning and belief revision is so close (cf. Makison and Gärdenfors 10]), preference relations are also relevant for belief revision.

The formal relationships we will describe are summarized in the following diagram.

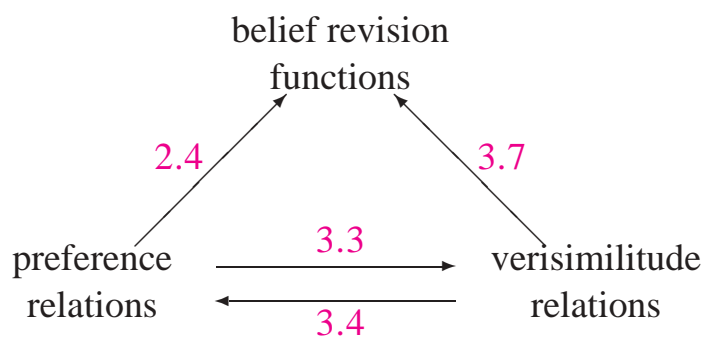


The nodes show the three topics which we interrelate. The arrows represent the definitions used to translate between the concepts, and are labelled by the definition number in the paper. For example, Definition 2.4 khows how to define a belief revision function from a preference relation.

The paper explores the properties of the translations. Firstly, we study how the postulates typically imposed on the three concepts fare under the translation mechanisms. Secondly, we examine under what conditions the above diagram commutes. For example, suppose we obtain a belief revision function from a preference relation by passing through a verisimilitude relation, using Definitions 3.3 and 3.7. Do we get the same result if we proceed directly, using Definition 2.49

We do not attempt a thorough review of any of the three relevant fields, such reviews being readily available elsewhere (e.g., Gärdenfors [4], Fuhrmann and Morreau [3] for belief revision, Brink [1], Kuipers 6] for verisimilitude, and [8] for preference relations). A brief introduction to verisimilitude is given in Section 3.

The paper is structured as follows. Section 2 discusses belief revision and preference relations. Section 3 introduces verisimilitude. Section 4 gives the results concerning the interrelationships between the conditions which can be imposed on preference relations and verisimilitude relations. Section 5 discusses under which circumstances the diagram commutes by exploring the compositions of the definitions. Finally, Section 6 draws conclusions.

Preliminaries We assume a language $\mathcal{L}$ which has the usual boolean connectives, a class $\mathcal{M}$ of interpretations of the language, and a relation $=$ in $\mathcal{M} \times \mathcal{L}$. We assume that $\models$ behaves classically with respect to the connectives. If $A \subseteq \mathcal{L}$ is a set of sentences, $\operatorname{Mod}(A)=\{m \in \mathcal{M} \mid \forall \psi \in A m \models \psi\}$. If $N \subseteq \mathcal{M}$, $\operatorname{Th}(N)=\{\varphi \in$ $\mathcal{L} \mid \forall m \in N m \models \varphi\}$. For $\varphi \in \mathcal{L}$ or $m \in \mathcal{M}$, we will write $\operatorname{Mod}(\varphi)$ and $\operatorname{Th}(m)$ instead of $\operatorname{Mod}(\{\varphi\})$ and $\operatorname{Th}(\{m\})$. The set $N \subseteq \mathcal{M}$ of interpretations is closed if $\operatorname{Mod}(\operatorname{Th}(N))=N$. The set $A \subseteq \mathcal{L}$ of sentences is closed if $\operatorname{Th}(\operatorname{Mod}(A))=A$. A closed set of sentences is also called a theory. The set of theories over $\mathcal{L}$ is $\mathcal{T}$. If $A \in \mathcal{T}, \operatorname{Ctg}(A)=\{B \in \mathcal{T} \mid A \subseteq B\}$ (the theories containing $A$ ). A theory $A$ is complete if $\varphi \in A$ or $\neg \varphi \in A$ for each $\varphi \in \mathcal{L}$; it is consistent if $\varphi \notin A$ or $\neg \varphi \notin A$ for each $\varphi \in \mathcal{L}$. The set of complete and consistent theories is denoted $C \mathcal{T}$.

We will need to make use of the fact that $m \in \operatorname{Mod}(\operatorname{Th}(n))$ iff $\operatorname{Th}(m)=\operatorname{Th}(n)$ iff $n \in \operatorname{Mod}(\operatorname{Th}(m))$. This is proved as follows. First assume $m \in \operatorname{Mod}(\operatorname{Th}(n))$; then, for all $\varphi \in \mathcal{L}, n \models \varphi$ implies $m \models \varphi$, $\operatorname{so} \operatorname{Th}(n) \subseteq \operatorname{Th}(m)$. Now suppose $n \not \models \varphi$; so $n \models \neg \varphi$, so $m \models \neg \varphi$, so $m \not \models \varphi$. Thus, $\operatorname{Th}(m) \subseteq \operatorname{Th}(n)$. This is the only place in the paper at which we appeal to the classical behaviour of $\models$. Now suppose $\operatorname{Th}(m)=\operatorname{Th}(n)$; then $n \models \varphi$ implies $m \models \varphi$, so $m \in \operatorname{Mod}(\operatorname{Th}(n))$. The other half is proved similarly.

If $\leq$ is a relation on the set $X$ and $Y \subseteq X$, then $y \in Y$ is said to be $\leq$-minimal in $Y$ if $\forall y^{\prime} \in Y\left(y^{\prime} \leq y \Rightarrow y \leq y^{\prime}\right)$. We define $\operatorname{Min}_{\leq}(Y)=\{y \in Y \mid y$ is $\leq$-minimal in $Y$. We define $\downarrow_{\leq} Y=\{x \in X \mid \exists y \in Y x \leq y\}$, and $\uparrow_{\leq} Y=\{x \in X \mid \exists y \in Y \quad y \leq x\}$. As usual, $x<y$ means $x \leq y$ and $y \not \leq x$.

2 Belief revision via preference relations The classical preference relations approach to default reasoning works as follows. Suppose $T$ is some default information expressed as sentences of the language $\mathcal{L}$. We assume some procedure for deriving 
from $T$ a relation $\sqsubseteq_{T} \subseteq \mathcal{M} \times \mathcal{M}$ which measures how nearly an interpretation $m$ satisfies the default information. By convention, $m \sqsubseteq_{T} n$ means that $m$ satisfies the default information as well as $n$ does; we say $m$ is preferred to $n$. There is a strong intuition that such preference relations should be transitive, though this is not always assumed in the literature. We will assume it. As to whether they are reflexive or not, this may be taken as a matter of convention for one can always close under reflexivity or take the strict (irreflexive) counterpart of a relation. We will assume reflexivity.

Definition 2.1 A preference relation $\sqsubseteq$ is a ternary relation $\sqsubseteq \subseteq \mathcal{M} \times \mathcal{T} \times \mathcal{M}$ such that, for all $T \in \mathcal{T}$, the binary relation $\sqsubseteq_{T}$ is reflexive and transitive.

There are several properties of preference relations which we will sometimes need. Some, such as stopperedness, are well known in the literature. Others, like the soundness property below, arise because we have made the parameter $T$ explicit.

Definition 2.2 A preference relation $\sqsubseteq$

1. is sound if for any satisfiable $T, m$ is $\sqsubseteq_{T}$-minimal in $\mathcal{M}$ iff $m \models T$.

2. is stoppered if for all $A \subseteq \mathcal{L}$ and $m \in \operatorname{Mod}(A)$ there is $n \in \operatorname{Min}_{\sqsubseteq_{T}}(\operatorname{Mod}(A))$ with $n \sqsubseteq_{T} m$.

3. is abstract if $\operatorname{Th}(m)=\operatorname{Th}(n)$ implies $m \sqsubseteq_{T} n$ and $n \sqsubseteq_{T} m$.

4. preserves closed sets if, for all $T \subseteq \mathcal{L}$ and closed $N \subseteq \mathcal{M}$, the sets $\operatorname{Min}_{\sqsubseteq_{T}}(N)$ and $\downarrow_{\sqsubseteq_{T}} N$ and $\uparrow_{\sqsubseteq_{T}} N$ are closed.

5. is strongly abstract if for all $N \subseteq \mathcal{M}$ and $m \in \operatorname{Mod}(\operatorname{Th}(N))$ we have $\exists n_{1}, n_{2} \in$ $N n_{1} \sqsubseteq_{T} m \sqsubseteq_{T} n_{2}$.

The intuition behind the soundness property is that nothing is "closer" to satisfying $T$ than its models. Stopperedness is well known in the default reasoning literature and tells us that we can find minimal models of any theory. Abstractness means that the preference of an interpretation is determined only by the sentences that it satisfies. The property of preservation of closed sets just tells us that certain useful operations on closed sets of interpretations return closed sets. Strong abstractness says that the preference order cannot make distinctions beyond the granularity of the logic. It represents an easy way to check whether the properties of abstractness and preservation of closed sets are satisfied, as the following lemma shows.

Lemma 2.3 If $\sqsubseteq$ is strongly abstract, it is abstract and preserves closed sets.

Proof: Abstractness:

$\operatorname{Th}(m)=\operatorname{Th}(n) \Rightarrow m \in \operatorname{Mod}(\operatorname{Th}(n))$

$\Rightarrow n \sqsubseteq_{T} m \sqsubseteq_{T} n$ (by strong abstractness).

Preservation of closed sets: Suppose $T \subseteq \mathcal{L}$ and $N \subseteq \mathcal{M}$ is closed. We prove that

1. $\operatorname{Min}_{\sqsubseteq_{T}}(N)$ is closed. Suppose $n \in \operatorname{Mod}\left(\operatorname{Th}\left(\operatorname{Min}_{\sqsubseteq_{T}}(N)\right)\right)$; we will prove that $n \in \operatorname{Min}_{\sqsubseteq_{T}}(N)$. By strong abstractness, there are $n_{1}, n_{2} \in \operatorname{Min}_{\bigsqcup_{T}}(N)$ such that $n_{1} \sqsubseteq_{T} n \sqsubseteq_{T} n_{2}$. Since $n_{1}$ and $n_{2}$ are both minimal and $n_{1} \sqsubseteq_{T} n_{2}$, we also have that $n_{2} \sqsubseteq_{T} n_{1}$. But $n_{1} \sqsubseteq_{T} n$, so by transitivity $n_{2} \sqsubseteq_{T} n$, i.e., they are all equivalent, so $n \in \operatorname{Min}_{\sqsubseteq_{T}}(N)$.

2. $\downarrow_{\sqsubseteq_{T}} N$ is closed. Suppose $n \in \operatorname{Mod}\left(\operatorname{Th}\left(\downarrow_{\sqsubseteq_{T}}(N)\right)\right)$; we prove $n \in \downarrow_{\sqsubseteq_{T}}(N)$. By strong abstractness, there are $n_{1}, n_{2} \in \downarrow_{\sqsubseteq_{T}}(N)$ such that $n_{1} \sqsubseteq_{T} n \sqsubseteq_{T} n_{2}$. Since 
$n_{2} \in \downarrow_{\sqsubseteq_{T}}(N)$, we have $n_{2} \sqsubseteq_{T} m$ for some $m \in N$; by transitivity, $n \sqsubseteq_{T} m$ and so $n \in \downarrow_{\bigsqcup_{T}}(N)$.

3. $\uparrow_{\sqsubseteq_{T}} N$ is closed: similar.

In the standard account of preference relations, $T$ is left implicit, and a fixed ordering $\sqsubseteq$ is assumed. This more general account presents $T$ as a parameter. In other work the authors and colleagues have described two preference structures; one based on the notion of "natural consequence" (as in Ryan [12]) and one based on distances between models (as in Ryan, Sernadas, and Sernadas [13]). In 15], Schobbens defines a preference structure for predicate logic based on correspondences.

Given a preference relation, we may define an inference relation. Let $A$ and $T$ be sets of sentences and $\varphi$ a sentence in $\mathcal{L}$. The inference relation $\sim \subseteq \mathcal{P}(\mathcal{L}) \times \mathcal{T} \times \mathcal{L}$ is defined as follows:

$$
A \sim_{T} \varphi: \Longleftrightarrow \operatorname{Min}_{\sqsubseteq T}(\operatorname{Mod}(A)) \subseteq \operatorname{Mod}(\varphi) .
$$

We will write $\psi \sim_{T} \varphi$ instead of $\{\psi\} \sim_{T} \varphi$. The preference relations framework has its origins in circumscription (cf. Lifschitz 7 ). Extensive work relating properties of $\bigsqcup_{T}$ to properties of $\sim_{T}$ can be found in the accounts of Makinson [9], Kraus, Lehmann, and Magidor [5], and Schlechta [14].

The connection established between default reasoning and belief revision reported in 10 seems to be essentially the following. The statement that $\psi \sim_{T} \varphi$ corresponds to the statement that $\varphi \in T * \psi$, i.e., that revising the information $T$ with $\psi$ will result in a theory that includes $\varphi$. In 10], the authors show that the relationship between the standard postulates for $\sim$ and for $*$ correspond very closely under this translation. Thus, a preference relation can be used to define a belief revision operator.

Definition $2.4(*$ in terms of $\sqsubseteq)$

$$
T *^{\sqsubseteq} \psi:=\operatorname{Th}\left(\operatorname{Min}_{\bigsqcup_{T}}(\operatorname{Mod}(\psi))\right) .
$$

This says that to revise $T$ with $\psi$, we look at the models of $\psi$ which are closest to $T$; then we take the theory of those models.

This definition is the first of the four definitions promised by the diagram in the introduction. Notice the notation: we write $* \sqsubset \sqsubset$ for the belief revision function defined in terms of the preference relation $\sqsubseteq$.

3 Verisimilitude via power orderings The topic of verisimilitude concerns the measurement of closeness of theories to the truth. The idea is to define a ternary relation on theories:

$$
A \leq_{T} B \text { if } A \text { is as close to } T \text { as } B \text { is. }
$$

Thus, the "truth" is represented as a theory. The actual truth is of course a complete theory (that is, for all $\varphi \in \mathcal{L}$, either $\varphi \in T$ or $\neg \varphi \in T$ ), but many of the definitions in the literature do not require this. Van Benthem's 18 constitutes a very readable survey of approaches as well as an analysis of the relations between verisimilitude and conditionals. We will again assume reflexivity and transitivity. 
Definition 3.1 A verisimilitude relation $\leq$ is a ternary relation in $\mathcal{T} \times \mathcal{T} \times \mathcal{T}$ such that, for all $T \in \mathcal{T}$, the binary relation $\leq_{T}$ is reflexive and transitive.

The ternary relation allows us to select, from a given range of theories, one which is closest to the truth in an obvious way: if the family $\left\{A_{i} \mid i \in I\right\}$ of theories consists of the candidates at hand, then a particular $A_{i}$ is closest to the truth if it is $\leq_{T}$-minimal in $\left\{A_{i} \mid i \in I\right\}$, i.e., $A_{i} \in \operatorname{Min}_{\leq_{T}}\left(\left\{A_{i} \mid i \in I\right\}\right)$. Of course there can be several incompatible theories among the candidates, all minimally close.

3.1 History of verisimilitude The first formal definition of this relation is due to Popper 11: for theories $A, B$, and $T$, he defines that $A \leq_{T}^{(P)} B$ iff $B \cap T \subseteq A$ and $A-T \subseteq B$. (The superscript $(P)$ stands for Popper.) Since $T$ contains only true sentences, the first condition in the definition can be thought of as saying that $A$ has all the true sentences that $B$ has. If $T$ is indeed complete, then its complement consists entirely of false sentences, in which case the second condition means that $A$ has no more false sentences in it than $B$ has. If $T$ is not complete then the second condition is not so intuitive.

Another definition of the same relation, due to Miller and Kuipers, is $A \leq_{T}^{(K)}$ $B$ if $\operatorname{Mod}(B) \cap \operatorname{Mod}(T) \subseteq \operatorname{Mod}(A)$ and $\operatorname{Mod}(A)-\operatorname{Mod}(T) \subseteq \operatorname{Mod}(B)$. We can paraphrase the two conditions as: any model in $B$ which might have been the true situation must also be a model in $A$ (so $A$ does not lose any models); and any model in $A$ which could not have been the true situation must be a model in $B$ (so $A$ does not introduce any bad models).

We have that

$$
A \leq_{T}^{(P)} B \text { implies } A \leq_{T}^{(K)} B,
$$

but the converse implication is false. It turns out that both Popper's definition and Miller and Kuiper's both have undesirable consequences. The following observations are due to Tichý and Miller:

\section{Proposition 3.2}

1. $A<_{T}^{(P)} B$ implies $A \subseteq T$.

2. If $T$ is complete and $\operatorname{Mod}(B) \cap \operatorname{Mod}(T)=\operatorname{Mod}(A)=\varnothing$ then $A \leq_{T}^{(K)} B$.

The first means that $\leq^{(P)}$ cannot strictly order "false" theories (that is, theories with at least one false sentence in them). Since that was the whole purpose of the enterprise, it seems sufficient reason to reject $\leq^{(P)}$. The second item in the proposition says that the contradictory theory $A$ (with no models) is an improvement on any theory $B$ which shares no models with $T$. It is counterintuitive that the contradictory theory should be an improvement on anything. A proof of the first item is given in Schurz and Weingartner [16], p. 49; the second is trivial to demonstrate. It should be noted that the second item is not seen as grounds for complete rejection of $\leq^{(K)}$; it is still widely discussed.

A survey of approaches to verisimilitude can be found in [1].

3.2 Power-ordering approach to verisimilitude The power-ordering approach to verisimilitude proceeds in the following way. We assume that $\mathcal{L}$ is propositional, and that the truth is a single interpretation $t$ in $\mathcal{M}$, or equivalently, that it is the complete 
theory $\mathrm{Th}(t)$. By convention, we take $t$ to be the interpretation in which every proposition is assigned true (we just rename the propositions to arrange this). The set $\mathcal{M}$ of interpretations has a natural order on it, given by $m \sqsubseteq_{t} n$ iff for all propositions $p$, $n \models p$ implies $m \models p$; which says, of course, that $m$ is as near to $t$ as $n$ is.

Thus, we have a natural order on interpretations which shows how they approximate the truth. We want an order on theories. Since a theory $T$ may be viewed as a set of interpretations (namely $\operatorname{Mod}(T)$ ), we may use a technique well known in computer science called the power-ordering or Egli-Milner ordering; it tells us how to lift a relation on points to sets of points. It says: if $R$ is a relation on $X$ then $R^{+}$is a relation on $\mathcal{P}(\mathcal{X})$, defined by $X R^{+} Y$ iff $\forall x \in X \exists y \in Y x R y \wedge \forall y \in Y \exists x \in X x R y$. Thus, Brink and Heidema define, for theories $A, B$,

$$
\begin{aligned}
A \leq_{t} B \Longleftrightarrow & \forall m \in \operatorname{Mod}(A) \exists n \in \operatorname{Mod}(B) m \sqsubseteq_{t} n \wedge \\
& \forall n \in \operatorname{Mod}(B) \exists m \in \operatorname{Mod}(A) m \sqsubseteq_{t} n .
\end{aligned}
$$

Further details and motivation are given in [2].

This approach is easily generalized. We need not assume that the "truth" is a complete theory (thus represented by a single model $t$ ), nor the particular ordering $\sqsubseteq_{t}$ given above. Indeed, we can start with any preference relation $\sqsubseteq$ and compute a corresponding verisimilitude relation:

Definition 3.3 ( $\leq$ in terms of $\sqsubseteq$ )

$$
\begin{aligned}
& A \leq \frac{\sqsubseteq}{T} B: \Longleftrightarrow \forall m \in \operatorname{Mod}(A) \exists n \in \operatorname{Mod}(B) m \sqsubseteq_{T} n \wedge \\
& \forall n \in \operatorname{Mod}(B) \exists m \in \operatorname{Mod}(A) m \sqsubseteq_{T} n \text {. }
\end{aligned}
$$

The intuition behind this definition is the following. The theory $A$ is as close to $T$ as $B$ is if every model of $A$ is as close to $T$ as some model of $B$ and also every model of $B$ is as far from $T$ as some model of $A$. Thus, $A$ is as close because it can match any model of $B$ with one of its models, and moreover, any of its models matches some $B$ model.

Conversely, starting with a verisimilitude relation (i.e., a ternary relation $\leq$ on theories) we can derive a family of relations on $\mathcal{M}$ via the "singleton embedding" of a relation in its power-relation.

Definition 3.4 ( $\sqsubseteq$ in terms of $\leq$ )

$$
m \sqsubseteq \stackrel{\leq}{T}: \Longleftrightarrow \operatorname{Th}(m) \leq_{T} \operatorname{Th}(n) .
$$

The intuition here is simple. Every interpretation $m$ gives us a theory, namely $\operatorname{Th}(m)$. If we're able to compare theories for closeness to $T$, then that fact allows us to compare interpretations too.

\section{Proposition 3.5}

1. If $\sqsubseteq$ is a preference relation, then $\leq \sqsubseteq$ is a verisimilitude relation.

2. If $\leq i$ a verisimilitude relation, then $\sqsubseteq \leq i$ is a preference relation.

Proof: 1. We just check that the power-relation of a pre-order is again a pre-order, a result known from the literature on power structures. Reflexivity is easy. For transitivity, suppose $A \leq_{T} B \leq_{T} C$; we will prove $A \leq_{T} C$. Suppose $m \in \operatorname{Mod}(A)$; since 
$A \leq_{T} B$ there exists $m^{\prime} \in \operatorname{Mod}(B)$ with $m^{\prime} \sqsubseteq_{T} m$. Using this $m^{\prime}$ and the fact that $B \leq_{T} C$, we find $n \in \operatorname{Mod}(C)$ with $n \sqsubseteq_{T} m^{\prime}$. By transitivity of $\Xi_{T} m \sqsubseteq_{T} n$. The proof of the other half is similar.

2. Reflexivity and transitivity of $\leq_{T}$ follow immediately from the reflexivity and transitivity of $\sqsubseteq_{T}$.

3.3 Conditions on verisimilitude relations A great many conditions on verisimilitude relations have been studied; for example, see 18 . However, the conditions that we will describe here seem to be yet more. The following conditions will be used in the remainder of the paper.

Definition 3.6 A verisimilitude relation $\leq$

1. is sound if for any satisfiable theory $T$, the theory $A$ is $\leq_{T}$-minimal in $\mathcal{T}$ iff $T \subseteq A$. This is the analogue of soundness for preference relations; it says the best theories are those that include the truth (and possibly more).

2. is stoppered if for all $A \subseteq \mathcal{L}$ and $B \in \operatorname{Ctg}(A)$ there is a satisfiable $C \in$ $\operatorname{Min}_{\leq_{T}}(\mathrm{Ctg}(A))$ with $C \leq_{T} B$; this is the natural analogue again.

3. respects complete theories if, for all $A \in \mathcal{T}$ and $B \in C \mathcal{T}, B \in \operatorname{Min}_{\leq_{T}}(\operatorname{Ctg}(A) \cap$ $C \mathcal{T}$ ) implies $B \in \operatorname{Min}_{\leq T}(\operatorname{Ctg}(A))$. This means that a complete theory which is minimal among the complete extensions of $A$ is also minimal among all the extensions.

4. is elaboration tolerant if, for $A, B, C \in \mathcal{T}, B \in \operatorname{Min}_{\leq T}(\operatorname{Ctg}(A))$ and $B \subseteq C$ imply $C \in \operatorname{Min}_{\leq T}(\operatorname{Ctg}(A))$. It says that if $B$ is closest to $T$ among the theories that contain $A$, then so is any elaboration of $B$.

5. satisfies split if

(a) $A \leq_{T} B$ and $\operatorname{Mod}(A)=\bigcup_{i \in I} \operatorname{Mod}\left(A_{i}\right)$ for some $I$ implies that there is a family $\left\{B_{i}\right\}_{i \in I}$ such that $\operatorname{Mod}(B)=\bigcup_{i \in I} \operatorname{Mod}\left(B_{i}\right)$ and $A_{i} \leq_{T} B_{i}$. This says that if $A \leq B$ and $A$ can be split into components $A_{i}$, then $B$ can be similarly split and each of the component pairs are related by $\leq_{T}$. Thus, a verisimilitude relation can be "split" into components.

(b) $A \leq_{T} B$ and $\operatorname{Mod}(B)=\bigcup_{i \in I} \operatorname{Mod}\left(B_{i}\right)$ for some $I$ implies that there is a family $\left\{A_{i}\right\}_{i \in I}$ such that $\operatorname{Mod}(A)=\bigcup_{i \in I} \operatorname{Mod}\left(A_{i}\right)$ and $A_{i} \leq_{T} B_{i}$. This is similar to (a).

6. satisfies join if $\operatorname{Mod}(A)=\bigcup_{i \in I} \operatorname{Mod}\left(A_{i}\right)$ and $\operatorname{Mod}(B)=\bigcup_{i \in I} \operatorname{Mod}\left(B_{i}\right)$ and $A_{i} \leq_{T} B_{i}$ for each $i \in I$, then $A \leq_{T} B$. This is the converse of split; it takes the components of a verisimilitude relation and "joins" them together.

7. is strongly abstract if for all sets of interpretations $N \subseteq \mathcal{M}$ and complete and consistent theories $A$ such that $\operatorname{Mod}(A) \subseteq \operatorname{Mod}(\operatorname{Th}(N))$, there exists $B_{1}, B_{2}$, complete, consistent, such that $B_{1} \leq_{T} A \leq_{T} B_{2}$ and $\operatorname{Mod}\left(B_{i}\right) \subseteq N$. This is a "literal translation" of strong abstractness for $\sqsubseteq$. It's a rather technical condition which we will need only once.

The analogues of the properties of preference relations are natural desiderata for verisimilitude. Note that there is no analogue of abstractness; every verisimilitude relation trivially has the property that $\operatorname{Mod}(A)=\operatorname{Mod}(B)$ implies $A \leq_{T} B$ and $B \leq_{T} A$. 
The other conditions given above may seem less natural, but it will be seen that they arise naturally from seeing verisimilitude in terms of the power-ordering construction.

3.4 Belief revision from verisimilitude We have so far defined a belief revision operator in terms of a preference relation (slightly generalizing the standard way; Definition 2.4], and we have shown how to define verisimilitude in terms of preference and vice versa (Definitions 3.3 and 3.4). We now complete the diagram in the introduction by giving a definition of belief revision in terms of verisimilitude.

To revise $T$ with $\psi$, we look at the theories which contain $\psi$ and pick among those the ones which are closest to $T$.

Definition $3.7(*$ in terms of $\leq)$

$$
T * \leq \psi:=\bigcap\left(\operatorname{Min}_{\leq T}(\operatorname{Ctg}(\psi))\right)
$$

Remark 3.8 The similarity in structure between Definitions 2.4 and 3.7 may be seen by the following:

- $T * \sqsubseteq \psi=\left\{\varphi \in \mathcal{L} \mid \operatorname{Min}_{\sqsubseteq_{T}}(\operatorname{Mod}(\psi)) \subseteq \operatorname{Mod}(\varphi)\right\}$;

- $T * \leq \psi=\left\{\varphi \in \mathcal{L} \mid \operatorname{Min}_{\leq_{T}}(\operatorname{Ctg}(\psi)) \subseteq \operatorname{Ctg}(\varphi)\right\}$.

We now have the following definitions to enable us to inter-define preference relations, verisimilitude relations and belief revision operators.

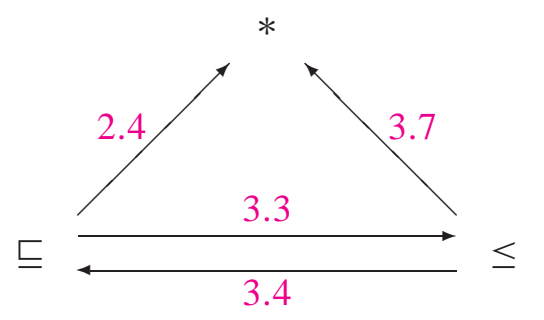

The remainder of the paper will explore properties of these definitions. First, in the next section, we examine the relations between the constraints given for preference relations and verisimilitude relations. We answer such questions as: what conditions must be imposed on $\leq$ in order to guarantee that $\sqsubseteq \leq$ is stoppered? Then, in the following section, we explore the round trips: what happens if we begin with a preference relation, convert it into a verisimilitude relation, and then back again into a preference relation? How do the two preference relations relate? The same question can be asked about the other way around, starting with a verisimilitude relation and doing a round trip via a preference relation. We also examine under what circumstances the two ways of defining belief revision coincide, i.e., when $* \leq=* \sqsubseteq$.

4 Interrelating the conditions In this section we examine how the conditions given for preference relations and verisimilitude relations translate using the definitions of $\sqsubseteq \leq$ and $\leq \sqsubseteq$.

We start with a lemma about Definition 3.3 which will be used often in this and the next section. It says that, in the power-ordering approach to verisimilitude, the theories closest to $T$ selected from the family of theories containing $B$ are precisely those whose models are closest to being models of $B$. 
Lemma 4.1 If $\sqsubseteq$ is stoppered and preserves closed sets, then

$$
B \in \operatorname{Min}_{\leq \frac{\bar{T}}{T}}(\operatorname{Ctg}(A)) \Longleftrightarrow \operatorname{Mod}(B) \subseteq \operatorname{Min}_{\sqsubseteq T}(\operatorname{Mod}(A)) .
$$

In other words, the best theories are those with the best models, "best" being relative to a given constraint.

Proof: $\quad$ Let $\leq$ be $\leq$ 드.

$\Rightarrow \quad$ Suppose $\operatorname{Mod}(B) \nsubseteq \operatorname{Min}_{\sqsubseteq_{T}}(\operatorname{Mod}(A))$. Then either $\operatorname{Mod}(B) \nsubseteq \operatorname{Mod}(A)$, i.e., $B \notin \mathrm{Ctg}(A)$, and we are home; or $B \in \mathrm{Ctg}(A)$ and $\exists n \in \operatorname{Mod}(B)-\operatorname{Min}_{\subseteq_{T}}(\operatorname{Mod}(A))$. In that case we will prove that $\operatorname{Th}\left(\downarrow_{\bigsqcup_{T}} \operatorname{Mod}(B) \cap \operatorname{Min}_{\bigsqcup_{T}}(\operatorname{Mod}(A))\right)<_{T} B$, thus proving that $B \notin \operatorname{Min}_{\leq T}(\operatorname{Ctg}(A))$.

1. We show $\leq_{T}$. If $m \in \operatorname{Mod}\left(\operatorname{Th}\left(\downarrow_{\sqsubseteq_{T}} \operatorname{Mod}(B) \cap \operatorname{Min}_{\sqsubseteq_{T}}(\operatorname{Mod}(A))\right)\right)$ then, since the sets $\downarrow_{\sqsubseteq_{T}} \operatorname{Mod}(B)$ and $\operatorname{Min}_{\sqsubseteq_{T}}(\operatorname{Mod}(A))$ and hence their intersection are all closed, $m \in \downarrow_{\sqsubseteq_{T}} \operatorname{Mod}(B)$. Therefore, $\exists n \in \operatorname{Mod}(B) m \sqsubseteq_{T} n$ as required. On the other hand, if $n \in \operatorname{Mod}(B)$ then $n \models A$, so by stopperedness we can find $m \in$ $\operatorname{Min}_{\sqsubseteq_{T}}(\operatorname{Mod}(A))$ with $m \sqsubseteq_{T} n$; and moreover, $m \in \downarrow_{\sqsubseteq_{T}} \operatorname{Mod}(B)$ as required.

2. We show $\nsucceq_{T}$. It is sufficient to show that there is some $n \models B$ such that, for all $m \models \operatorname{Th}\left(\downarrow_{\sqsubseteq_{T}} \operatorname{Mod}(B) \cap \operatorname{Min}_{\sqsubseteq_{T}}(\operatorname{Mod}(A))\right), n \nsubseteq_{T} m$. Take any $n \in \operatorname{Mod}(B)-$ $\operatorname{Min}_{\sqsubseteq_{T}}(\operatorname{Mod}(A))$.

$\Leftarrow$ Suppose $B \notin \operatorname{Min}_{\leq T}(\operatorname{Ctg}(A))$. Then either $B \notin \operatorname{Ctg}(A)$, so $\operatorname{Mod}(B) \nsubseteq \operatorname{Mod}(A)$, or $\operatorname{Mod}(B) \subseteq \operatorname{Mod}(A)$ and $C<_{T} B$ for some $C \in \operatorname{Ctg}(A)$. In the former case we are home. In the latter, we must find $n \in \operatorname{Mod}(B)-\operatorname{Min}_{\sqsubseteq_{T}}(\operatorname{Mod}(A))$. We have

1. $\forall c \models C \exists b \models B c \sqsubseteq_{T} b$;

2. $\forall b \models B \exists c \models C c \sqsubseteq_{T} b$; and

3. $\exists c \models C \forall b \models B b \nsubseteq_{T} c \vee \exists b \models B \forall c \models C b \Phi_{T} c$.

If we have the first disjunct of 3 , take this $c$. By 1 , find $b ; c \sqsubseteq_{T} b$, so by 3 again $c \sqsubset_{T} b$ and so $b \notin \operatorname{Min}_{\sqsubseteq_{T}}(\operatorname{Mod}(A))$. If we had the second disjunct of 3, take this $b$. Take $c$ from line 2. Again, $c \sqsubset_{T} b$. Therefore, $b \notin \operatorname{Min}_{\bigsqcup_{T}}(\operatorname{Mod}(A))$, $\operatorname{so} \operatorname{Mod}(B) \nsubseteq$ $\operatorname{Min}_{\sqsubseteq_{T}}(\operatorname{Mod}(A))$.

Now we give our main result for this section, relating the properties of $\sqsubseteq$ and $\leq$.

\section{Proposition 4.2}

1. If $\sqsubseteq$ preserves closed sets, then $\leq \sqsubseteq$ satisfies split.

2. $\leq \sqsubseteq$ satisfies join.

3. If $\sqsubseteq$ is abstract, then $\leq \sqsubseteq$ respects complete theories.

4. If $\sqsubseteq$ is sound, stoppered and preserves closed sets then $\leq \sqsubseteq$ is sound.

5. If $\sqsubseteq$ is stoppered, then $\leq \sqsubseteq$ is stoppered and elaboration-tolerant.

6. If $\sqsubseteq$ is strongly abstract, then so is $\leq \sqsubseteq$.

7. If $\leq i$ sound and respects complete theories, then $\sqsubseteq \leq i$ sound.

8. If $\leq$ is stoppered, elaboration tolerant, and satisfies split, then $\sqsubseteq \leq i$ is stoppered.

9. $\sqsubseteq \leq$ is abstract.

10. If $\leq$ is strongly abstract, then so is $\sqsubseteq \leq$. 


\section{Proof:}

1. If $A \leq_{T} B$ and $\operatorname{Mod}(A)=\bigcup_{i \in I} \operatorname{Mod}\left(A_{i}\right)$ then define $B_{i}=\operatorname{Th}\left(\uparrow_{\subseteq_{T}} \operatorname{Mod}\left(A_{i}\right) \cap\right.$ $\operatorname{Mod}(B))$. First we show $\operatorname{Mod}(B)=\bigcup_{i \in I} \operatorname{Mod}\left(B_{i}\right)$, i.e.,

$$
\operatorname{Mod}(B)=\bigcup_{i \in I} \operatorname{Mod}\left(\operatorname{Th}\left(\uparrow_{\subseteq_{T}} \operatorname{Mod}\left(A_{i}\right) \cap \operatorname{Mod}(B)\right)\right) .
$$

$\subseteq$ : If $b \models B$ then there exists $a \models A$ with $a \sqsubseteq_{T} b$. Let $i$ be such that $a \in$ $\operatorname{Mod}\left(A_{i}\right)$. Then $b \in \uparrow_{\sqsubseteq_{T}} \operatorname{Mod}\left(A_{i}\right) \cap \operatorname{Mod}(B), \operatorname{so} b \in \operatorname{Mod}\left(\operatorname{Th}\left(\uparrow_{\sqsubseteq_{T}} \operatorname{Mod}\left(A_{i}\right) \cap\right.\right.$ $\operatorname{Mod}(B)))$ for that $i$.

$\supseteq$ : For any $i$ we have that $\uparrow_{\sqsubseteq_{T}} \operatorname{Mod}\left(A_{i}\right) \cap \operatorname{Mod}(B) \subseteq \operatorname{Mod}(B)$, and therefore $\operatorname{Mod}\left(\operatorname{Th}\left(\uparrow_{\subseteq_{T}} \operatorname{Mod}\left(A_{i}\right) \cap \operatorname{Mod}(B)\right)\right) \subseteq \operatorname{Mod}(\operatorname{Th}(\operatorname{Mod}(B)))=\operatorname{Mod}(B), \operatorname{prov}-$ ing the result.

Next we prove that $A_{i} \leq_{T} B_{i}$. Suppose $a \in \operatorname{Mod}\left(A_{i}\right)$; then $a \in \operatorname{Mod}(A)$, so pick $b \in \operatorname{Mod}(B)$ such that $a \sqsubseteq_{T} b$. Then $b \in \uparrow_{\sqsubseteq_{T}} \operatorname{Mod}\left(A_{i}\right) \cap \operatorname{Mod}(B)$. On the other hand, suppose $b \in \operatorname{Mod}\left(B_{i}\right)$; then since $\operatorname{Mod}\left(A_{i}\right)$ and $\operatorname{Mod}(B)$ are closed and $\sqsubseteq$ preserves closed sets, $\uparrow_{\sqsubseteq_{T}} \operatorname{Mod}\left(A_{i}\right) \cap \operatorname{Mod}(B)$ is closed. Hence, $b \in \uparrow_{\sqsubseteq_{T}} \operatorname{Mod}\left(A_{i}\right) \cap \operatorname{Mod}(B)$, so take $a \in \operatorname{Mod}\left(A_{i}\right)$ such that $a \sqsubseteq_{T} b$.

2. Suppose $\operatorname{Mod}(A)=\bigcup_{i \in I} \operatorname{Mod}\left(A_{i}\right)$ and $\operatorname{Mod}(B)=\bigcup_{i \in I} \operatorname{Mod}\left(B_{i}\right)$ and $A_{i} \leq_{T}$ $B_{i}$ for each $i \in I$. We want to prove that $A \leq_{T} B$. Suppose $a \in \operatorname{Mod}(A)$; then $a \in \operatorname{Mod}\left(A_{i}\right)$ for some $i$, and since $A_{i} \leq_{T} B_{i}$ there is a $b \in \operatorname{Mod}\left(B_{i}\right) \subseteq \operatorname{Mod}(B)$ with $a \sqsubseteq_{T} b$. Similarly, given $b \in \operatorname{Mod}(B)$ we can find $a \in \operatorname{Mod}(A)$ with $a \sqsubseteq_{T}$ $b$.

3. Suppose $A \in \mathcal{T}$ and $B \in C \mathcal{T}, B \in \operatorname{Min}_{\leq \frac{\bar{T}}{T}}(\operatorname{Ctg}(A) \cap C \mathcal{T})$. We will show $B \in$ $\operatorname{Min}_{\leq \frac{\ulcorner}{T}}(\operatorname{Ctg}(A))$. Suppose not. Clearly, $B \in \operatorname{Ctg}(A)$; suppose $C<\frac{5}{T} B$. Our reasoning now is similar to the proof of Lemma 4.1. we have

(a) $\forall c \models C \exists b \models B c \sqsubseteq_{T} b$;

(b) $\forall b \models B \exists c \models C c \sqsubseteq_{T} b$; and

(c) $\exists c \models C \forall b \models B b \unrhd_{T} c \vee \exists b \models B \forall c \models C b \unrhd_{T} c$.

From these facts we find $c \sqsubset_{T} b$ for some $b \models B, c \models C$, and hence, employing abstractness, $\operatorname{Th}(c)<\frac{\bar{T}}{T} \operatorname{Th}(b)$. Since $B$ is complete, $B=\operatorname{Th}(b)$ and therefore, $B \notin \operatorname{Min}_{\leq \frac{\ulcorner}{T}}(C t g(A) \cap C \mathcal{T})$, a contradiction.

4. $\sqsubseteq$ sound $\Leftrightarrow \operatorname{Mod}(T)=\operatorname{Min}_{\sqsubseteq T}(\mathcal{M})$

$\Rightarrow \quad \forall A\left(\operatorname{Mod}(A) \subseteq \operatorname{Mod}(T) \Leftrightarrow \operatorname{Mod}(A) \subseteq \operatorname{Min}_{\sqsubseteq_{T}}(\mathcal{M})\right)$

$\Leftrightarrow \quad \forall A\left(A \in \operatorname{Ctg}(T) \Leftrightarrow A \in \operatorname{Min}_{\leq \frac{5}{T}}(\operatorname{Ctg}(\varnothing))\right)$

$\Leftrightarrow \operatorname{Ctg}(T)=\operatorname{Min}_{\leq \frac{\bar{T}}{T}}(\mathcal{T})$

$\Leftrightarrow \quad \leq \subseteq$ sound.

In going from the second line to the third, we use Lemma 4.1.

5. $\leq$ 드 is stoppered: Suppose $B \in \mathrm{Ctg}(A)$. We want a $C \in \operatorname{Min}_{\leq \frac{5}{T}}(\operatorname{Ctg}(A))$ with $C \leq \overline{\bar{T}} B$. Put $C=\operatorname{Th}\left(\downarrow_{\sqsubseteq_{T}} \operatorname{Mod}(B) \cap \operatorname{Min}_{\sqsubseteq_{T}}(\operatorname{Mod}(A))\right)$. $C$ is satisfiable because $\downarrow_{\sqsubseteq_{T}} \operatorname{Mod}(B) \cap \operatorname{Min}_{\sqsubseteq_{T}}(\operatorname{Mod}(A)) \neq \varnothing$. The fact that $C \leq \frac{\bar{T}}{T} B$ follows easily; to prove $C \in \operatorname{Min}_{\leq \frac{\bar{T}}{T}}(\operatorname{Ctg}(A))$, use Lemma 4.1. 
$\leq=$ elaboration-tolerant: Suppose $A, B, C$ theories with $B \in \operatorname{Min}_{\leq \frac{\ulcorner}{T}}(\operatorname{Ctg}(A))$ and $B \subseteq C$. We want to prove $C \in \operatorname{Min}_{\leq \frac{c}{T}}(\operatorname{Ctg}(A))$.

$$
\begin{aligned}
B & \in \operatorname{Min}_{\leq \frac{\subseteq}{T}}(\operatorname{Ctg}(A)) \wedge B \subseteq C \\
& \Leftrightarrow \operatorname{Mod}(B) \subseteq \operatorname{Min}_{\subseteq_{T}}(\operatorname{Mod}(A)) \wedge \operatorname{Mod}(C) \subseteq \operatorname{Mod}(B) \\
& \Rightarrow \operatorname{Mod}(C) \subseteq \operatorname{Min}_{\sqsubseteq_{T}}(\operatorname{Mod}(A)) \\
& \Leftrightarrow C \in \operatorname{Min}_{\leq \frac{\ulcorner}{T}}(\operatorname{Ctg}(A))
\end{aligned}
$$

6. Suppose $N$ and $A$ are given as in the definition of strong abstractness for $\leq$. Pick $m \in \operatorname{Mod}(A)$. Using the fact that $\sqsubseteq$ is strongly abstract, pick $n_{1}, n_{2}$ with $n_{1} \sqsubseteq_{T} m \sqsubseteq_{T} n_{2}$ and $n_{1}, n_{2} \in N$. Then $\operatorname{Th}\left(n_{1}\right) \leq \frac{\sqsubseteq}{T} A \leq \frac{\sqsubseteq}{T} \operatorname{Th}\left(n_{2}\right)$, since $\operatorname{Th}(m)=$ $A$. Since $\sqsubseteq$ is abstract, $\operatorname{Mod}\left(\operatorname{Th}\left(n_{i}\right)\right) \subseteq N$.

7. $T \subseteq B \Leftrightarrow B \in \operatorname{Min}_{\leq_{T}}(\mathcal{T}) \quad \leq$ sound

$\Rightarrow\left(T \subseteq \operatorname{Th}(m) \Leftrightarrow \operatorname{Th}(m) \in \operatorname{Min}_{\leq_{T}}(\mathcal{T})\right) \quad$ in particular

$\Rightarrow\left(T \subseteq \operatorname{Th}(m) \Leftrightarrow \operatorname{Th}(m) \in \operatorname{Min}_{\leq_{T}}(C \mathcal{T})\right) \quad$ respects complete theories

$\Rightarrow\left(m \in \operatorname{Mod}(T) \Leftrightarrow m \in \operatorname{Min}_{\sqsubseteq \leq}(\mathcal{M})\right) \quad$ def. of $\sqsubseteq \leq$

8. Let $A$ be a set of sentences such that $m \models A$. We require that $n \sqsubseteq \leq m$ with $n \in \operatorname{Min}_{\sqsubseteq \leq}^{\leq}(\operatorname{Mod}(A))$. $\operatorname{Th}(m) \in \operatorname{Ctg}(A)$, so since $\leq$ is stoppered we can find a satisfiable $B \in \operatorname{Min}_{\leq_{T}}(\operatorname{Ctg}(A))$ with $B \leq \operatorname{Th}(m)$. Pick any $n \models B$. By elaboration tolerance, $\operatorname{Th}(n) \in \operatorname{Min}_{\leq_{T}}(\operatorname{Ctg}(A))$. Moreover, since $\operatorname{Mod}(\operatorname{Th}(n)) \subseteq$ $\operatorname{Mod}(B)$ we have $\operatorname{Mod}(B)=\operatorname{Mod}(\operatorname{Th}(n)) \cup \operatorname{Mod}(B)$. Using this fact and $B \leq_{T} \operatorname{Th}(m)$, split $\operatorname{Mod}(\operatorname{Th}(m))$ into two subsets, $\operatorname{Mod}\left(A_{1}\right)$ and $\operatorname{Mod}\left(A_{2}\right)$ with $\operatorname{Th}(n) \leq_{T} A_{1}$. But $A_{1}$ is either $\operatorname{Th}(m)$ or $\mathcal{L}$, since $\operatorname{Th}(m)$ is complete. The latter situation is impossible because of the case $I=\varnothing$ of split, and so we have $\operatorname{Th}(n) \leq_{T} \operatorname{Th}(m)$, i.e., $n \sqsubseteq \frac{\leq}{T} m$. A similar argument shows that $n \in$ $\operatorname{Min}_{\sqsubseteq \frac{\leq}{T}}(\operatorname{Mod}(A))$.

9. Suppose $m, n \in \mathcal{M}$ such that $\operatorname{Th}(m)=\operatorname{Th}(n)$. Then $\operatorname{Th}(m) \leq_{T} \operatorname{Th}(n)$ (reflexivity), so $m \sqsubseteq \frac{\leq}{T} n$.

10. If $m \in \operatorname{Mod}(\operatorname{Th}(N))$, then $\operatorname{Mod}(\operatorname{Th}(m)) \subseteq \operatorname{Mod}(\operatorname{Th}(N))$. Pick $B_{1}, B_{2}$ by strong abstractness of $\leq$. Pick $n_{1}, n_{2}$ in $\operatorname{Mod}\left(B_{1}\right), \operatorname{Mod}\left(B_{2}\right)$ respectively. Then $n_{i} \in N$ and $\operatorname{Th}\left(n_{i}\right)=B_{i}$, each $i$, so $n_{1} \sqsubseteq \frac{\leq}{T} m \sqsubseteq n_{2}$.

5 Composing the definitions In this section we look at whether the diagram given in the introduction commutes. First we consider a round trip: suppose we begin with a preference relation, calculate the verisimilitude relation according to Definition 3.3, and return to a preference relation via Definition 3.4. Intuitively we expect to arrive back at the same preference relation, since the verisimilitude relation contains much more structure than a preference relation. A verisimilitude relation contains information about partial, incomplete situations whereas a preference relation just orders (total) models. Going from $\sqsubseteq$ to $\leq$ freely generates a particular "canonical" ordering of theories, which from the point of view of $\sqsubseteq$ contains a lot of redundancy. Going the other way forgets this extra structure.

Indeed, it is sufficient to impose the relatively benign condition of abstractness on $\sqsubseteq$ in order to guarantee that the round trip preserves the preference relation. 
Proposition 5.1 If $\sqsubseteq$ is abstract, $\sqsubseteq^{\leq}=\sqsubseteq$.

Proof:

$$
\begin{aligned}
m \sqsubseteq_{T}^{\leq} n \Leftrightarrow & \operatorname{Th}(m) \leq \bar{\complement} \operatorname{Th}(n) \\
\Leftrightarrow & \forall m^{\prime} \models \operatorname{Th}(m) \exists n^{\prime} \models \operatorname{Th}(n) m^{\prime} \sqsubseteq T n^{\prime} \wedge \\
& \forall n^{\prime} \models \operatorname{Th}(n) \exists m^{\prime} \models \operatorname{Th}(m) m^{\prime} \sqsubseteq T n^{\prime} .
\end{aligned}
$$

From the Introduction, $m^{\prime} \models \operatorname{Th}(m)$ implies $\operatorname{Th}\left(m^{\prime}\right)=\operatorname{Th}(m)$, which, since $\sqsubseteq$ is abstract, means that $m^{\prime} \sqsubseteq_{T} n^{\prime}$ iff $m \sqsubseteq_{T} n^{\prime}$. By a similar argument, this reduces to $m \sqsubseteq_{T} n$, and the result is proved.

The other round-trip is less well behaved. If we go from a verisimilitude relation to a preference relation and then back again, there is no guarantee that we will recover the original verisimilitude relation. The intuitive reason has already been stated: the verisimilitude relation contains a lot of structure, which is jettisoned by Definition 3.4 and then a canonical version of which is freely generated by Definition 3.3. However, we should expect that the round-trip will preserve the relation for complete theories.

Proposition 5.2 If $A, B$ are complete and consistent theories and $\leq i$ is abstract, $A \leq \overline{\bar{T}}^{\leq} \mathrm{B}$ iff $A \leq_{T} B$.

Proof: $\quad$ Since $\leq$ is abstract, so is $\sqsubseteq \leq$. Since $A$ is complete and consistent, it is equal to $\operatorname{Th}(m)$ for some $m$ (indeed, any $m \in \operatorname{Mod}(A)$ ). Similarly, $B=\operatorname{Th}(n)$ for some $n$.

$$
\begin{aligned}
A \leq \overline{\bar{T}}^{\leq} B \Leftrightarrow & \forall m^{\prime} \models \operatorname{Th}(m) \exists n^{\prime} \models \operatorname{Th}(n) m^{\prime} \sqsubseteq n_{T} \\
& \wedge \forall n^{\prime} \models \operatorname{Th}(n) \exists m^{\prime} \models \operatorname{Th}(m) m^{\prime} \sqsubseteq \leq n_{T}^{\prime} \\
\Leftrightarrow & m \sqsubseteq \frac{\leq}{T} \\
\Leftrightarrow & \operatorname{Th}(m) \leq T \operatorname{Th}(n) \\
\Leftrightarrow & A \leq_{T} B
\end{aligned}
$$

The reasoning from the second formulation to the third uses the fact that $\sqsubseteq \leq$ is abstract in a similar way to the proof of the previous proposition.

We may formulate some conditions on $\leq$ which will guarantee that the roundtrip via a preference will return exactly the same verisimilitude relation. These conditions are rather strong, forcing $\leq$ to order incomplete situations in a way compatible with the way it orders complete ones. This is the role of the conditions split and join in the following proposition. The requirement of strong abstractness is there for the technical reason that it guarantees that certain sets are closed.

Proposition 5.3 If $\leq$ is strongly abstract and satisfies split and join, then $\leq^{\sqsubseteq}=\leq$.

Proof: Note that $\sqsubseteq \leq$ is strongly abstract (Proposition 4.2 5 ) and therefore it is abstract and preserves closed sets (Lemma 2.3). We want to show $A \leq_{T} B$ iff: $\forall a \in$ $\operatorname{Mod}(A) \exists b \in \operatorname{Mod}(B) \operatorname{Th}(a) \leq_{T} \operatorname{Th}(b)$ and $\forall b \in \operatorname{Mod}(B) \exists a \in \operatorname{Mod}(A) \operatorname{Th}(a) \leq_{T}$ $\operatorname{Th}(b)$.

$\Leftarrow$ : $\quad$ Let $I$ be the disjoint union of $\operatorname{Mod}(A)$ and $\operatorname{Mod}(B)$. If $i$ is some $a$ in $\operatorname{Mod}(A)$, let $A_{i}=\operatorname{Th}(a)$ and $B_{i}=\operatorname{Th}(b)$ where $b$ is the $b$ which comes from $a$ using the RHS. Similarly, if $i$ is some $b$ in $\operatorname{Mod}(B)$, let $B_{i}=\operatorname{Th}(b)$ and $A_{i}=\operatorname{Th}(a)$ where $a$ is the $a$ which comes from $b$ using the RHS. Then $\operatorname{Mod}(A)=\bigcup_{i \in I} \operatorname{Mod}\left(A_{i}\right)$ and $\operatorname{Mod}(B)=$ $\bigcup_{i \in I} \operatorname{Mod}\left(B_{i}\right)$ and $A_{i} \leq_{T} B_{i}$, so by join $A \leq_{T} B$. 
$\Rightarrow$ : We have $A \leq_{T} B$, and want to show the RHS. For the first part of the RHS: we suppose $a \in \operatorname{Mod}(A)$, and want to find $b \in \operatorname{Mod}(B)$ with $\operatorname{Th}(a) \leq_{T} \operatorname{Th}(b)$.

Consider $\operatorname{Mod}(B)=\bigcup_{n \in \operatorname{Mod}(B)}\{n\} ;$ by split, find the family $\left\{A_{n} \mid n \in \operatorname{Mod}(B)\right\}$ which covers $A$, i.e., $\operatorname{Mod}(A)=\bigcup_{n \in \operatorname{Mod}(B)} \operatorname{Mod}\left(A_{n}\right)$. Since $a \in \operatorname{Mod}(A)$, pick $b$ such that $a \in \operatorname{Mod}\left(A_{b}\right)$. By the use of split we have $A_{b} \leq_{T} \operatorname{Th}(b)$.

Now use split again, this time writing $\operatorname{Mod}\left(A_{b}\right)=\bigcup_{m \in \operatorname{Mod}\left(A_{b}\right)}\{m\}$, to find the family $\left\{B_{m}\right\}$ such that $\operatorname{Mod}(\operatorname{Th}(b))=\bigcup_{m \in \operatorname{Mod}\left(A_{b}\right)} \operatorname{Mod}\left(B_{m}\right)$ and for each $m \in$ $\operatorname{Mod}\left(A_{b}\right)$, we have $\operatorname{Th}(m) \leq_{T} B_{m}$. In particular, $\operatorname{Th}(a) \leq_{T} B_{a}$. But each such $B_{m}$ is equal either to $\operatorname{Th}(b)$ or to $\mathcal{L}$, since $\operatorname{Mod}\left(B_{m}\right)$ is a subset of the models of $\operatorname{Th}(b)$ all of which are satisfaction-equivalent. Thus, either $\operatorname{Th}(a) \leq_{T} \operatorname{Th}(b)$ or $\operatorname{Th}(a) \leq_{T} \mathcal{L}$. The latter case is ruled out by the special case $I=\varnothing$ of split, so we are left with $\operatorname{Th}(a) \leq_{T} \operatorname{Th}(b)$.

The second part of the RHS is similar.

Finally, we ask: when do $* \leq$ and $* \subseteq$ coincide? This is the other aspect of the question of whether the diagram commutes.

Proposition 5.4 If $\sqsubseteq$ is stoppered and preserves closed sets then $* \sqsubseteq=*^{\leq} \leq \underline{\square}$.

Proof: We show that $\operatorname{Min}_{\subseteq_{T}}(\operatorname{Mod}(\psi)) \subseteq \operatorname{Mod}(\varphi)$ iff $\operatorname{Min}_{\leq_{T}}(\operatorname{Ctg}(\psi)) \subseteq \operatorname{Ctg}(\varphi)$.

$\Rightarrow$ : Suppose $C \in \operatorname{Min}_{\leq T}(\operatorname{Ctg}(\psi))$. We need to prove $C \in \operatorname{Ctg}(\varphi)$. By Lemma 4.1. $\operatorname{Mod}(C) \subseteq \operatorname{Min}_{\subseteq_{T}}(\operatorname{Mod}(\psi)), \operatorname{so} \operatorname{Mod}(C) \subseteq \operatorname{Mod}(\varphi)$, so $C \in \operatorname{Ctg}(\varphi)$.

$\Leftarrow: \quad$ Suppose $N \subseteq \operatorname{Min}_{\sqsubseteq_{T}}(\operatorname{Mod}(\psi))$. We need to prove $N \subseteq \operatorname{Mod}(\varphi)$. Let $B=$ $\operatorname{Th}(N)$. Then, since $\operatorname{Min}_{\sqsubseteq_{T}}(\operatorname{Mod}(\psi))$ is closed, $\operatorname{Mod}(B) \subseteq \operatorname{Min}_{\sqsubseteq_{T}}(\operatorname{Mod}(\psi))$, so by Lemma 4.1. $B \in \operatorname{Min}_{\leq_{T}}(\operatorname{Ctg}(A))$. Therefore $B \in \operatorname{Ctg}(\varphi)$, so $N=\operatorname{Mod}(B) \subseteq$ $\operatorname{Mod}(\varphi)$.

The conditions required for this proposition are relatively weak, as one might expect, in view of the fact that verisimilitude relations potentially contain more information than preference relations, but those verisimilitude relations which are generated from a preference relation do not contain any surprises. The conditions required in the next proposition are stronger, because we have to constrain the verisimilitude relation more.

Proposition 5.5 If $\leq$ respects complete theories and is elaboration-tolerant then $* \leq=*^{\underline{I}} \leq$.

Proof: It is sufficient to prove $\operatorname{Min}_{\leq_{T}}(\psi) \subseteq \operatorname{Ctg}(\varphi)$ iff $\operatorname{Min}_{\leq_{T}}(\operatorname{Ctg}(\psi) \cap C \mathcal{T}) \subseteq$ $\operatorname{Ctg}(\varphi) \cap C \mathcal{T}$.

$\Rightarrow$ : This follows easily from the fact that $\leq$ respects complete theories.

$\Leftarrow$ : Suppose $C \in \operatorname{Min}_{\leq_{T}}(\operatorname{Ctg}(\psi))$. We want to prove that $\operatorname{Mod}(C) \subseteq \operatorname{Mod}(B)$. Take $m \models C$. By the fact that $\leq$ is elaboration-tolerant, $\operatorname{Th}(m) \in \operatorname{Min}_{\leq_{T}}(\operatorname{Ctg}(A))$; so $\operatorname{Th}(m) \in \operatorname{Ctg}(B)$, i.e., $m \models B$.

6 Conclusions and outlook We have given an intuitive definition of belief revision in terms of verisimilitude and shown close connections between the preferential models approach to belief revision and the power-ordering approach to verisimilitude. The connection may be succinctly summarized as follows. 
Preference relations order models according to how close they are to some given theory, whereas verisimilitude relations order theories according to the same criterion. We have shown how to extract a verisimilitude relation from a preference relation and vice-versa, and we have shown sufficient conditions to prove that the notions are inter-definable. In general, verisimilitude relations contain more information than preference relations because they say how to order partial theories as well as total models. Moving from a verisimilitude relation to a preference relation discards this extra information, whereas moving in the opposite direction freely generates a canonical version of it.

Further work will complete the triangle of Section 1 by finding definitions of a preference relation and verisimilitude relation in terms of an arbitrary belief revision operator. Of course, a definition of $\sqsubseteq$ in terms of $*$ already exists in the literature, by going via the non-monotonic inference operator $\sim$ and using the representation theorems of 5 and others. Therefore, we can also construct $\leq$ by applying Definition 3.3 but more likely, there is a more interesting way of constructing $\leq$ directly from $*$ which exploits the extra freedom of a verisimilitude relation.

Acknowledgments The first author is grateful to Chris Brink and the other organizers for their invitation to the UNISA/UCT Verisimilitude Workshop (7-11 February 1994, Pretoria, South Africa) during which he began working on these ideas. Both authors are grateful to Sjoerd Zwart and to an anonymous referee for their comments.

This work is related to the authors' participation in the Esprit WG ModelAge and MCM Medicis, and the first author's participation in the Esprit WG IS-CORE and the Portuguese JNICT project JURAD. We acknowledge our colleagues in those projects for interesting discussions and the funding agencies for financial assistance.

\section{REFERENCES}

[1] Brink, C., "Verisimilitude: views and reviews," History and Philosophy of Logic, vol. 10 (1989), pp. 181-201.Zbl 0674.03002 MR 90k:03003 1.3.1

[2] Brink, C., and J. Heidema, "A verisimilar ordering of theories based in a propositional language," The British Journal for the Philosophy of Science, vol. 38 (1987), pp. 533549. 3.2

[3] Fuhrmann, A., and M. Morreau, editors, The Logic of Theory Change, number 465 in Lecture Notes in Artificial Intelligence, Springer-Verlag, Berlin, 1991. Zbl 0874.03007 1

[4] Gärdenfors, P., Knowledge in Flux, MIT Press, Cambridge, 1988. MR 89k:00015 1

[5] Kraus, S., D. Lehmann, and M. Magidor, "Non-monotonic reasoning, preferential models and cumulative logics," Artificial Intelligence, vol. 44 (1990), pp. 167-207. 1.|2.6

[6] Kuipers, T., editor, What is Closer-to-the-Truth, Rodopi, Amsterdam, 1987.

[7] Lifschitz, V., "Circumscription," pp. 297-352 in Handbook of Logic in Artificial Intelligence, edited by D. Gabbay, C. Hogger, and J. Robinson, Oxford University Press, Oxford, 1994. MR 95k:68213 2

[8] Makinson, D., "General theory of cumulative inference," pp. 1-18 in Non-monotonic Reasoning, edited by M. Reinfrank, J. de Kleer, and M. L. Ginsberg, Lecture Notes in Artificial Intelligence 346, Springer-Verlag, Berlin, 1988. Zbl 0675.03007 MR 90f:03051 III 
[9] Makinson, D., "General patterns in non-monotonic reasoning," pp. 35-110 in Handbook of Logic in Artificial Intelligence, edited by D. Gabbay, C. Hogger, and J. Robinson, Oxford University Press, Oxford, 1994. 2

[10] Makinson, D., and P. Gärdenfors, "Relations between the logic of theory change and non-monotonic logic," pp. 185-205 in The Logic of Theory Change, edited by A. Fuhrmann and M. Morreau, Lecture Notes in Artificial Intelligence 465, SpringerVerlag, Berlin, 1991. Zbl 0925.03130|MR 1096795 1 1.12.2.2

[11] Popper, K. R., Conjectures and Refutations, Routledge and Kegan Paul, London, 1963. \begin{tabular}{|l|l|}
\hline MR 27:2393 3.1 \\
\hline
\end{tabular}

[12] Ryan, M. D., "Defaults and revision in structured theories," pp. 362-373 in Proceedings of the Sixth IEEE Symposium on Logic in Computer Science (LICS), IEEE Computer Society Press, Los Alamitos, 1991.

[13] Ryan, M. D., A. Sernadas, and C. Sernadas, "Adjunctions between default frameworks," in Information Systems: Correctness and Reuse, edited by R. Wieringa and R. Feenstra, World Scientific Publishing, Singapore, 1995. 2

[14] Schlechta, K., "Some results on classical preferential models," Journal of Logic and Computation, vol. 2 (1992), pp. 675-686. Zbl 0785.03014 MR 94f:68168|2 2

[15] Schobbens, P.-Y., "Exceptions for algebraic specifications: on the meaning of 'but'," Science of Computer Programming, vol. 20 (1993), pp. 73-111.Zbl 0783.68085 MR 94j:68205 2

[16] Schurz, G., and P. Weingartner, "Verisimilitude defined by relevant consequence elements," pp. 47-78 in What is Closer-to-the-Truth, edited by T. Kuipers, Rodopi, Amsterdam, 1987. 3.1

[17] Shoham, Y., "A semantical approach to nonmonotonic logics," pp. 388-392 in Proceedings of the 10th International Conference on Artificial Intelligence (IJCAI), Morgan Kaufmann, San Mateo, 1987.

[18] van Benthem, J., "Verisimilitude and conditionals," pp. 103-128 in What is Closer-tothe-Truth, edited by T. Kuipers, Rodopi, Amsterdam, 1987. 3,3.3

School of Computer Science

University of Birmingham

Edgbaston, Birmingham B15 2TT

U.K.

email: M.D.Rvan@cs.bham.ac.uk

Institut d'Informatique

Fac. Univ. Notre-Dame de la Paix

Rue Grandgagnage 21

5000 Namur

Belgium

email: pvs@info.fundp.ac.be 